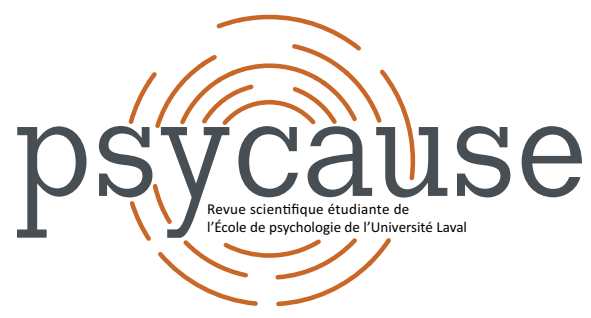

\title{
PSYCAUSE
}

Revue scientifique étudiante de l'École de psychologie de l'Université Laval

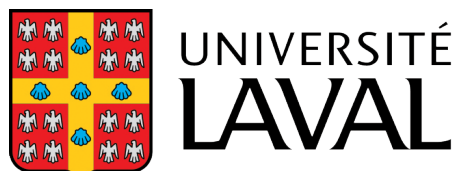

Faculté des sciences sociales École de psychologie

NOVEMBRE 2020 - VOL. $10 \mathrm{~N}^{\circ} 2$

\section{EFFETS DE LA MARCHE ATTENTIVE SUR DES VARIABLES PSYCHO-COGNITIVES CHEZ LES BACHELIERS}

Elizabeth POULIN ${ }^{1}$, Léonie PROULX ${ }^{1}$, Catherine BÉGIN ${ }^{1}$ \& Sonia GOULET ${ }^{1}$

1 École de psychologie, Université Laval

*elizabeth.poulin.3@ulaval.ca

\section{Pour citer l'article}

Poulin, E., Proulx, L., Bégin, C., \& Goulet, S. (2020). Effets de la marche attentive sur des variables psycho-cognitives chez les bacheliers. Psycause: Revue scientifique étudiante de l'École de psychologie de I'Université Laval, 10(2), 7-8. 


\title{
EFFETS DE LA MARCHE ATTENTIVE SUR DES VARIABLES PSYCHO- COGNITIVES CHEZ LES BACHELIERS
}

\author{
Elizabeth POULIN¹, Léonie PROULX'1, Catherine BÉGIN \& Sonia GOULET ${ }^{1}$ \\ ${ }^{1}$ École de psychologie, Université Laval \\ *elizabeth.poulin.3@ulaval.ca
}

\section{Mots-clés : Marche attentive, marche consciente, co-marche dialoguée, pleine conscience}

La pleine conscience (PC) est la capacité à maintenir délibérément l'attention sur un objet dans le moment présent et sans jugement (Kabat-Zinn, 2004). Les pratiques formelles de PC (p. ex., méditation assise sur le souffle) présentent des obstacles pouvant compromettre l'adhérence et l'assiduité aux exercices, tels que l'engourdissement mental, les douleurs posturales, etc. D'autre part, les pratiques de PC informelles consistent à être pleinement conscient dans les activités de la vie quotidienne (Birtwell, Williams, van Marwijk, Armitage, \& Sheffield, 2019). Pratiquer la PC conjointement à une activité physique améliorerait la santé et le bien-être (Yang \& Conroy, 2018). Toutefois, il existe peu de preuves scientifiques rigoureuses des bienfaits de la PC informelle isolée des autres pratiques (Zhang, 2018). Ainsi, la marche attentive autoguidée sur la base d'instructions écrites apparaît comme une forme prometteuse d'entraînement autonome informel à la PC. L'étude vise à tester la faisabilité et les effets d'un programme bref de marche attentive (MA) sur des variables psycho-cognitives. L'étude compare les effets de la MA avec ceux d'une condition de co-marche dialogique (CM) et de marche non dirigée contrôle (C) sur la PC, la flexibilité psychologique, le bien-être mental ainsi que la conscience intéroceptive $(\mathrm{Cl})$ et corporelle (CC) chez un échantillon non clinique de bacheliers'. L'objectif premier est d'évaluer dans quelle mesure la MA influence la PC chez une population non clinique d'étudiants universitaires comparativement à d'autres modalités de marche. Les objectifs secondaires sont de mettre en lumière les relations entre les variables identifiées comme concordantes ou contraires à un niveau optimal de PC.

\section{Méthode}

Dix-neuf étudiants de premier cycle de I'Université Laval inscrits à temps plein sont recrutés et prennent part à un programme de marche. Les participants sont assignés aléatoirement à trois groupes: MA $(N=7), \mathrm{CM}(N=6)$ et $\mathrm{C}$ $(N=6)$. Les données avant le programme de marche (prétest) et après celui-ci (posttest) sont recueillies par le biais de questionnaires auto-rapportés: la Mindful Attention Awareness Scale - Adaptation française; The Acceptance and Action Questionnaire for University Students (AAQ-US); L'Échelle de bien-être mental de Warwick-Edinburgh; The
Multidimensional Assessment of Interoceptive Awareness Version 2 (MAIA-2). Trois versions d'un journal de bord sont élaborées pour les groupes MA, CM et $C$ et doivent être remplis après chaque pratique. Ces programmes s'échelonnent sur trois semaines à raison de trois pratiques de 20 minutes par semaine.

\section{Résultats et discussion}

À la lumière des résultats issus du test du $U$ de Mann-Whitney (Tableau 1), aucune différence intergroupe n'est notée sur les moyennes des pentes calculées entre le pré-test et le posttest à l'échelle de PC. Toutefois, la mesure de CC diffère entre les groupes $M A$ et $C M$, mais ne diffère pas entre les groupes MA et C. II n'existe pas de différence significative entre les moyennes des pentes sur la mesure de la PC entre le groupe $\mathrm{CM}$ et $\mathrm{C}$, tandis qu'une différence significative émerge entre la moyenne des pentes sur la mesure de la CC entre MA et CM. De nombreuses associations positives sont observées entre les variables d'intérêt en prétest (Tableau 2) et en posttest (Tableau 3).

De nombreuses interprétations sont sujettes à l'explication d'absence d'effets significatifs sur la PC et les variables dépendantes à l'étude, notamment les caractéristiques de l'échantillon (petite taille échantillonnale), la présence de possibles variables modératrices (discours interne des universitaires), le manque de sensibilité de la mesure de marche non dirigée supplémentaire et l'équivalence imparfaite des groupes en pré-test.

Selon ces résultats, il est impossible de conclure que le programme de MA a des effets positifs sur la PC. La majorité des résultats sont non significatifs, malgré des tailles d'effet modérées à fortes, sauf pour la variable de PC entre les groupes contrôles ( $\mathrm{CM}$ et $\mathrm{C}$ ) où une taille d'effet faible à modérée est observée. Chez une population non clinique de bacheliers, la MA serait bénéfique à la CC en comparaison à un groupe de marche non dirigée $(C)$, où l'évolution à cette variable pré-test posttest est significative avec une forte taille d'effet. II est attendu que cette étude permette d'obtenir des indications sur les forces et les obstacles liés à l'implantation de programmes de PC informelle en

1 Dans ce présent rapport de recherche, l'emploi du masculin pour désigner les participants n'a d'autres fins que celle d'alléger le texte. 
milieu universitaire, en plus d'étayer les connaissances sur la marche attentive, les pratiques de PC informelle et la
$\mathrm{Cl}$ en PC informelle afin de guider l'élaboration de futurs programmes de PC.

Tableau 1

Résultats aux questionnaires selon le groupe de marche et le temps de mesure

\begin{tabular}{|c|c|c|c|c|c|c|c|c|c|c|}
\hline \multirow{3}{*}{$\begin{array}{l}\text { Groupes } \\
\text { MA }(\mathrm{N}=7)\end{array}$} & \multicolumn{10}{|c|}{ Questionnaires } \\
\hline & \multicolumn{2}{|c|}{ MAAS } & \multicolumn{2}{|c|}{ AAQ-US } & \multicolumn{2}{|c|}{ WEMWBS } & \multicolumn{2}{|c|}{ MAIA-Cl } & \multicolumn{2}{|c|}{ MAIA-CC } \\
\hline & $\mathrm{T} 1$ & $\mathrm{~T} 2$ & $\mathrm{~T} 1$ & $\mathrm{~T} 2$ & T1 & T2 & $\mathrm{T} 1$ & $\mathrm{~T} 2$ & $\mathrm{~T} 1$ & T2 \\
\hline$M$ & 64,86 & 62,43 & 67,29 & 62,29 & 52,57 & 56,14 & 97,29 & 100,29 & 7,00 & 5,43 \\
\hline ET & 11,80 & 7,46 & 8,69 & 13,02 & 5,35 & 6,36 & 17,97 & 16,93 & 4,40 & 4,28 \\
\hline$M$ pentes & \multicolumn{2}{|c|}{$-2,43$} & \multicolumn{2}{|c|}{$-5,00$} & \multicolumn{2}{|c|}{3,57} & \multicolumn{2}{|c|}{3,00} & \multicolumn{2}{|c|}{$-1,57$} \\
\hline ET pentes & \multicolumn{2}{|c|}{15,09} & \multicolumn{2}{|c|}{12,75} & \multicolumn{2}{|c|}{7,61} & \multicolumn{2}{|c|}{21,13} & \multicolumn{2}{|c|}{8,44} \\
\hline $\mathrm{CM}(\mathrm{n}=6)$ & T1 & $\mathrm{T} 2$ & $\mathrm{~T} 1$ & T2 & T1 & T2 & $\mathrm{T} 1$ & $\mathrm{~T} 2$ & T1 & $\mathrm{T} 2$ \\
\hline M & 57,83 & 61,67 & 62,00 & 66,33 & 53,67 & 53,83 & 101,00 & 109,17 & 5,67 & 8,00 \\
\hline ET & 6,31 & 7,50 & 7,59 & 9,69 & 2,34 & 3,31 & 15,77 & 9,66 & 2,66 & 2,04 \\
\hline$M$ pentes & \multicolumn{2}{|c|}{3,83} & \multicolumn{2}{|c|}{4,33} & \multicolumn{2}{|c|}{0,17} & \multicolumn{2}{|c|}{8,17} & \multicolumn{2}{|c|}{3,67} \\
\hline ET pentes & \multicolumn{2}{|c|}{11,39} & \multicolumn{2}{|c|}{14,99} & \multicolumn{2}{|c|}{5,31} & \multicolumn{2}{|c|}{14,77} & \multicolumn{2}{|c|}{3,08} \\
\hline$C(N=6)$ & $\mathrm{T} 1$ & $\mathrm{~T} 2$ & T1 & $\mathrm{T} 2$ & T1 & $\mathrm{T} 2$ & $\mathrm{~T} 1$ & $\mathrm{~T} 2$ & $\mathrm{~T} 1$ & $\mathrm{~T} 2$ \\
\hline$M$ & 54,5 & 63,17 & 58,17 & 63,00 & 50,83 & 47,50 & 101,50 & 100,67 & 5,5 & 7,33 \\
\hline ET & 12,13 & 12,88 & 9,81 & 14,45 & 6,08 & 9,40 & 19,33 & 23,39 & 4,40 & 4,28 \\
\hline$M$ pentes & \multicolumn{2}{|c|}{8,67} & \multicolumn{2}{|c|}{4,83} & \multicolumn{2}{|c|}{$-3,33$} & \multicolumn{2}{|c|}{$-0,83$} & \multicolumn{2}{|c|}{2,67} \\
\hline ET pentes & \multicolumn{2}{|c|}{14,28} & \multicolumn{2}{|c|}{22,06} & \multicolumn{2}{|c|}{13,88} & & & & \\
\hline
\end{tabular}

Note. La MAAS et le AAQ-US présentent des résultats inversés pour fins de comparaison.

$N$ : nombre de participants par groupe; $M$ : moyenne; ET: écart-type; $M$ des pentes: moyenne des pentes; ET pentes: écart-type des pentes; T1: Temps 1; et T2: Temps 2

Tableau 2

Corrélations entre les cinq variables en prétest

\begin{tabular}{lccccc}
\hline & 1 & 2 & 3 & 4 & 5 \\
\hline 1. MAAS & 1 & - & - & - & - \\
2. AAQ-US & $0,612^{\star \star}$ & 1 & - & - & - \\
3. WEMWBS & 0,053 & $0,586^{\star \star}$ & 1 & - & - \\
4. MAIA-CI & 0,165 & 0,269 & 0,199 & 1 & - \\
5. MAIA-CC & 0,362 & 0,307 & 0,247 & $0,643^{\star \star}$ & 1 \\
\hline
\end{tabular}

Note. ${ }^{\star \star} p<0,001$ (2-tailed); ${ }^{*} p<0,05$ (2-tailed).

\section{Références}

Birtwell, K., Williams, K., van Marwijk, H., Armitage, C. J., \& Sheffield, D. (2019). An exploration of formal and informal mindfulness practice and associations with wellbeing. Mindfulness, 10(1), 89-99. https://doi.org/10.1007/s12671018-0951-y

Kabat-Zinn, J. (2004). Wherever you go, there you are: Mindfulness meditation for everyday life. Londres: Piatkus Books Ltd.

\section{Pour citer l'article}

Poulin, E., Proulx, L., Bégin, C., \& Goulet, S. (2020). Effets de la marche attentive sur des variables psycho-cognitives chez les bacheliers. Psycause: Revue scientifique étudiante de l'École de psychologie de l'Université Laval, 10(2), 7-8.
Tableau 3

Corrélations entre les cinq variables en posttest

\begin{tabular}{lccccc}
\hline & 1 & 2 & 3 & 4 & 5 \\
\hline 1. MAAS & 1 & - & - & - & - \\
2. AAQ-US & $0,727^{\star \star}$ & 1 & - & - & - \\
3. WEMWBS & $0,642^{\star \star}$ & $0,632^{\star \star}$ & 1 & - & - \\
4. MAIA-CI & 0,328 & $0,513^{\star}$ & $0,495^{\star}$ & 1 & - \\
5. MAIA-CC & $-0,159$ & $-0,132$ & $-0,226$ & 0,370 & 1 \\
\hline
\end{tabular}

Note. ${ }^{* \star} p<0,001$ (2-tailed) ; ${ }^{\star} p<0,05$ (2-tailed).

Yang, C. H., \& Conroy, D. E. (2018). Momentary negative affect is lower during mindful movement than while sitting: An experience sampling study. Psychology of Sport and Exercise, 37, 109-116. https://doi.org/10.1016/j.psychsport.2018.05.003

Zhang, K. (2018). Identifying effective informal mindfulness practices in daily activities. Journal of International Buddhist Studies (JIBS), 9(2), 1-18. Repéré sur http://www. ojs.mcu.ac.th 